\title{
Dynamical behaviors of an HTLV-I infection model with intracellular delay and immune activation delay
}

\author{
Jinliang Wang ${ }^{1}$, Kaifa Wang ${ }^{2 *}$ and Zhichao Jiang ${ }^{3}$
}

"Correspondence:

kfwang72@163.com

${ }^{2}$ School of Biomedical Engineering,

Third Military Medical University,

Chongqing, 400038, P.R. China

Full list of author information is

available at the end of the article

\begin{abstract}
This paper investigates the dynamics of an HTLV-I infection model with intracellular delay and immune activation delay. The primary objective of the study is to consider the effect of the time delay on the stability of the infected equilibrium. Two sharp threshold parameters $\Re_{0}$ and $\Re_{1}$ are identified as the basic reproduction number for viral infection and for CTLs response, respectively, which determine the long time behaviors of the viral infection. In particular, our mathematical analysis reveals that a Hopf bifurcation occurs when immune activation delay passes through a critical value. Using the normal form theory and center manifold arguments, the explicit formulae which determine the stability, the direction, and the period of bifurcating periodic solutions are derived. Numerical simulations are given to support the theoretical results.
\end{abstract}

MSC: $34 \mathrm{C} 05 ; 92 \mathrm{D} 25$

Keywords: HTLV-l; intracellular delay; immune activation delay; stability; Hopf bifurcation

\section{Introduction}

Human T-cell leukaemia/lymphoma virus type I (HTLV-I) is a retrovirus infecting primarily $\mathrm{CD} 4^{+} \mathrm{T}$ cells, and the transmission occurs remarkably through direct cell-to-cell contact. It is reported that HTLV-I associated myelopathy/tropical spastic paraparesis (HAM/TSP) patients harbor higher proviral loads in peripheral blood lymphocytes than asymptomatic carriers [1]. Also, a remarkable amount of circulating HTLV-I specific CD8 ${ }^{+}$ cytotoxic T lymphocytes (CTLs) circulates in the peripheral blood of HAM/TSP patients present. It is convincing that the persistent cytotoxicity of the CTLs is the reason for the development of a progressive neurologic disease, i.e., HAM/TSP [2, 3], and several HTLVI-associated diseases.

Understanding the role played by the CTLs in controlling the HTLV-I infection is vital to identifying risk factors for the development of HAM/TSP. Several mathematical models have investigated the dynamics of the interaction in vivo among HTLV-I, the CD4 $4^{+}$target cells, and the CTLs immune response in order to explain the pathogenesis of HTLV-Iassociated diseases [4-10] and used as a tool to study the role of immune response in the viral dynamics. It is advocated that time delays cannot be ignored in models for im-

(c) 2015 Wang et al. This article is distributed under the terms of the Creative Commons Attribution 4.0 International License (http://creativecommons.org/licenses/by/4.0/), which permits unrestricted use, distribution, and reproduction in any medium, provided you give appropriate credit to the original author(s) and the source, provide a link to the Creative Commons license, and indicate if changes were made. 
mune response [9]. Recently, HTLV-I infection models given by systems of delay differential equations (DDEs) have been studied by several authors, who analyzed the effect of delay in the local and global dynamics of the model, bifurcations, and several rich dynamical behaviors (see, e.g., $[1,10-15]$ and the references cited therein).

Denote by $x(t), y(t), z(t)$ the concentrations of uninfected, infected, and HTLV-I-specific $\mathrm{CD} 8^{+}$CTLs at time $t$, respectively. In [9], Wang et al. incorporated a time delay into the immune response, $z^{\prime}(t)=c y(t-\omega)-b z(t)$, where the CTLs response is activated at ate proportional to the number of infected cells at a previous time, $c y(t-\omega)$, and it also decays exponentially at a rate proportional to its current strength $b z$. Numerical results reveal that stability switches, periodic solutions, and chaotic solutions can be observed. Time delay is commonly incorporated to account for a series of immunological events leading to the CTLs response, and it may arise through a number of different processes. In [15, 16], the authors explored the effect of HTLV-I interaction through a system of delay differential equations (DDEs). The delayed CTLs immune response takes the following form: $z^{\prime}(t)=c y(t-\omega) z(t-\omega)-b z(t)$, where the CTLs response activated at time $t$ is proportional to the product of the amount of CTLs at $t-\omega$ and that of infected cells at $t-\omega$. It is shown through numerical simulations that delayed CTLs response can lead to sustained oscillations through a Hopf bifurcation.

Recently, HTLV-I model with time delays in the CTL response leading to the coexistence of multiple stable periodic solutions has been studied in [17]. These multiple stable periodic solutions differ in amplitude and period and have their own basins of attraction. In [18], by taking the immune delay as a bifurcation parameter, $\mathrm{Xu}$ and Wei theoretically proved the global existence of multiple periodic solutions in HTLV-I infection model with CTL immune response.

Because the principle of CTLs activation by infected cells is complex and is not known clearly, in the line of work of Huang et al. [19], we shall investigate the case where CTLs are stimulated at time $t$ proportional to the product of the amount of CTLs at $t$ and that of infected cells at $t-\omega$, which can be described in the following way:

$$
z^{\prime}(t)=c y(t-\omega) z(t)-b z(t) .
$$

Biologically, (1.1) represents that the immune system needs some time to develop a suitable response after the recognition of non-self cells.

In present paper, to further account for the latent period for the cell to cell infection, we assume that virus transmission occurs after the virus entry with a constant time lag $\tau>0$. Here, the intracellular delay $\tau$ describes the latent period between the time when target cells are infected and the time when infected cells start producing virions to integration, i.e., $\mathrm{CD} 4^{+} \mathrm{T}$ cells infected at time $t$ will be activated at time $t+\tau$. The number of actively infected target cells at time $t$ is given by a delayed mass-action (bilinear incidence) term $\beta e^{-d \tau} x(t-\tau) y(t-\tau)$, where $e^{-d \tau}$ describes the probability of infected target cells surviving the period of intracellular delay from $t-\tau$ to $t$. Constant $d$ denotes the death rate for infected cells (but not yet virus producing cells).

Denote by $\lambda$ the recruitment rate of healthy $\mathrm{CD} 4^{+} \mathrm{T}$ cells. CTL-driven elimination of infected $\mathrm{CD} 4^{+} \mathrm{T}$ cells is assumed to be of the form $\gamma y z$, where constant $\gamma$ accounts for the strength of CTLs elimination. Denote by $d_{1}, d_{2}, d_{3}$ the turnover rates of uninfected, infected $\mathrm{CD} 4^{+} \mathrm{T}$, and $\mathrm{CD} 8^{+} \mathrm{CTLs}$, respectively. The preceding assumptions lead to the 
following HTLV-I infection model with delayed cell to cell infection and CTLs response:

$$
\left\{\begin{array}{l}
x^{\prime}(t)=\lambda-d_{1} x(t)-\beta x(t) y(t) \\
y^{\prime}(t)=\beta e^{-d \tau} x(t-\tau) y(t-\tau)-d_{2} y(t)-\gamma y(t) z(t), \\
z^{\prime}(t)=\mu y(t-\omega) z(t)-d_{3} z(t)
\end{array}\right.
$$

All parameters in system (1.2) are assumed to be positive. The initial conditions of system

(1.2) at $t=0$ are given as

$$
\varphi=\left(\varphi_{1}, \varphi_{2}, \varphi_{3}\right) \in \mathcal{C}^{+}, \quad \varphi(0)>0,
$$

where $\mathcal{C}^{+}$denotes the Banach space of continuous real-valued functions $\mathcal{C}=\mathcal{C}\left([-\sigma, 0], \mathbb{R}_{+}^{3}\right)$ with the sup-norm

$$
\|\varphi\|=\max \left\{\sup _{-\sigma \leq \theta \leq 0}\left|\varphi_{1}(\theta)\right|, \sup _{-\sigma \leq \theta \leq 0}\left|\varphi_{2}(\theta)\right|, \sup _{-\sigma \leq \theta \leq 0}\left|\varphi_{3}(\theta)\right|\right\}
$$

for $\sigma=\max \{\tau, \omega\}$.

The aim of the present paper is to carry out a complete mathematical analysis of dynamic behaviors of system (1.2) and find out the different influences between intracellular delay and immune activation delay. The paper is organized as follows. In Section 2, we present preliminary results of system (1.2), including positivity and boundedness of solutions, the existence of equilibria, and the definition of the basic reproduction numbers for viral infection $\left(\Re_{0}\right)$ and for CTLs response $\left(\Re_{1}\right)$. Section 3 is devoted to the global dynamics of system (1.2) when $\Re_{1} \leq 1$. Using the two key threshold parameters $\Re_{0}$ and $\Re_{1}$, we establish the global dynamics of system (1.2) by the techniques of Lyapunov functionals. When $\Re_{1}>1$, Section 4 first gives the global dynamics of system (1.2) in the case where $\tau$ is present and $\omega$ is absent. The results indicate that the intracellular delay does not affect the stability of the system. Thus, we can neglect the intracellular delay $\tau$ in system (1.2). Therefore, we identify parameter regimes in which immune activation delay $\omega$ can destabilize the HAM/TSP equilibrium and lead to a Hopf bifurcation. Using the normal form theory and center manifold argument, the explicit formulae which determine the stability and direction of bifurcated periodic solutions are derived. Numerical simulations are carried out to explain the mathematical conclusions in Section 5. The last section ends with a summary and discussion.

\section{Preliminaries}

Proposition 2.1 Under initial conditions in (1.3), all solutions of system (1.2) are positive and ultimately bounded in $\mathcal{C}$.

Proof By the existence and uniqueness theorem (Theorem 2.1 of Kuang [20]) of DDEs, there exists $t_{0}>0$ such that there exists a solution $(x(t), y(t), z(t))$ of system (1.2) for $0<t<$ $t_{0}$. We assume that there exists a solution of system (1.2) for $0<t<t_{1}$ for positive $t_{1}$, where the existence is assured by the theorem stated above.

First, we prove that $x(t)$ is positive for all $t \geq 0$. Assuming the contrary and letting $t_{1}>0$ be the first time such that $x\left(t_{1}\right)=0$, we have $x^{\prime}\left(t_{1}\right)=\lambda>0$ by the first equation of system (1.2). Hence $x(t)<0$ for $t \in\left(t_{1}-\varepsilon, t_{1}\right)$, where $\varepsilon>0$ is a sufficiently small constant. This contradicts $x(t)>0$ for $t \in\left[0, t_{1}\right)$. It follows that $x(t)>0$ for $t>0$ as long as $x(t)$ exists. 
By the second equation of system (1.2), we have

$$
y(t)=y(0) e^{-d_{2} t-\gamma \int_{0}^{t} z(\theta) d \theta}+\int_{0}^{t} \beta e^{-d \tau} x(\theta-\tau) y(\theta-\tau) e^{d_{2}(\theta-t)} e^{-\gamma \int_{\theta}^{t} z(\sigma) d \sigma} d \theta .
$$

It follows that $y(t)>0$ for $t>0$.

From the third equation of system (1.2), we have

$$
z(t)=z(0) e^{\int_{0}^{t}\left(\mu y(\theta-\omega)-d_{3}\right) d \theta} .
$$

This shows that $z(t) \geq 0$ for $0 \leq t<t_{1}$ and that $z(t)$ is bounded as $t$ tends to $t_{1}$, because $\theta-\omega \leq t_{1}-\omega$. If $z(0)>0$, then $z(t)>0$ for $0 \leq t<t_{1}$ by (2.2).

Next we show that positive solutions of (1.2) are ultimately uniformly bounded for $t \geq 0$. Put

$$
K(t)=e^{-d \tau} x(t)+y(t+\tau) .
$$

Adding all the equations of (1.2) we get

$$
\begin{aligned}
K^{\prime}(t) & =\lambda e^{-d \tau}-d_{1} e^{-d \tau} x(t)-d_{2} y(t+\tau)-\gamma y(t+\tau) z(t+\tau) \\
& \leq \lambda e^{-d \tau}-\ell K(t),
\end{aligned}
$$

where $\ell=\min \left\{d_{1}, d_{2}\right\}$. There exist $M_{2}>0$ and $T_{1}>0$ such that $y(t) \leq M_{2}$ for each $t \geq T_{1}$. For example, we can take $M_{2}=\frac{\lambda e^{-d \tau}}{\ell}$. If $t>T_{1}+\tau+\omega$, we have

$$
\begin{aligned}
z(t) & =z(t-\omega) \exp \left(\mu \int_{t-\omega}^{t} y(\theta-\omega) d \theta-b \omega\right) \\
& \leq e^{\mu M_{2} \omega} z(t-\omega) .
\end{aligned}
$$

We put

$$
G(t)=e^{-d \tau} x(t)+y(t+\tau)+\frac{\gamma}{\mu} e^{-\mu M_{2} \omega} z(t+\tau+\omega) .
$$

Adding all the equations of (1.2) we get

$$
\begin{aligned}
G^{\prime}(t)= & \lambda e^{-d \tau}-d_{1} e^{-d \tau} x(t)-d_{2} y(t+\tau)-\gamma y(t+\tau) z(t+\tau) \\
& +\frac{\gamma}{\mu} e^{-\mu M_{2} \omega}\left[\mu y(t+\tau) z(t+\tau+\omega)-d_{3} z(t+\tau+\omega)\right] \\
\leq & \lambda e^{-d \tau}-d G(t),
\end{aligned}
$$

where $d=\min \left\{d_{1}, d_{2}, d_{3}\right\}$. This shows that there exists $T$ with $T>T_{1}+\tau+\omega$ and $M_{1}>0$ such that $G(t) \leq M_{1}$ for each $t \geq T$. Each solution is contained in the bounded domain $\Gamma=\left\{(x, y, z) \in \mathbb{R}^{3} \mid 0 \leq x \leq M_{1}, 0 \leq y \leq M_{1}, 0 \leq z \leq M_{1}\right\}$ for sufficiently large $t$, which does not depend on the initial conditions. 
System (1.2) always has an infection-free equilibrium $E_{0}=\left(x_{0}, 0,0\right)$, where $x_{0}=\lambda / d_{1}$, which means that the infected cells are cleared. The basic reproductive number of the viruses for system (4.1) is given by

$$
\Re_{0}=\frac{\lambda \beta e^{-d \tau}}{d_{1} d_{2}} .
$$

This number describes the average number of newly generated infected cells from one infected cell at the beginning of the infection process. When $\Re_{0}>1$, in addition to $E_{0}$, the system can have two chronic-infection equilibria $E_{1}=\left(x_{1}, y_{1}, 0\right)$ and $E_{2}=\left(x_{2}, y_{2}, z_{2}\right)$ in $\Gamma$, where

$$
x_{1}=\frac{d_{2} e^{d \tau}}{\beta}, \quad y_{1}=\frac{d_{1}}{\beta}\left(\Re_{0}-1\right) .
$$

At equilibrium $E_{1}$, the HTLV-I infection is persistent with a constant proviral load $y_{1}>0$, whereas the CTLs response is absent, which means that there is the risk for developing HAM/TSP. This corresponds to the situation of an asymptotic carrier.

$$
x_{2}=\frac{\lambda \mu}{\mu d_{1}+\beta d_{3}}=\frac{d_{2} \Re_{1} e^{d \tau}}{\beta}, \quad y_{2}=\frac{d_{3}}{\mu}, \quad z_{2}=\frac{d_{1} d_{2} \mu+\beta d_{2} d_{3}}{\gamma\left(\mu d_{1}+\beta d_{3}\right)}\left(\Re_{1}-1\right)
$$

where

$$
\Re_{1}=\frac{\lambda \beta \mu e^{-d \tau}}{d_{1} d_{2} \mu+\beta d_{2} d_{3}} .
$$

Therefore, $E_{2}$ exists in the interior of $\Gamma$ if and only if $\Re_{1}>1$. At equilibrium $E_{2}$, both the proviral load and CTLs response persist at a constant level. This corresponds to the situation of a HAM/TSP patient. By aforementioned analysis, we obtain that the existence of equilibria of (4.1) depends only on two threshold parameters $\left(\Re_{0}\right.$ and $\left.\Re_{1}\right)$, which are called the basic reproduction numbers for viral infection and for CTLs response, respectively.

Note that

$$
\Re_{1}=\frac{\lambda \mu \beta e^{-d \tau}}{d_{1} d_{2} \mu+\beta d_{2} d_{3}}=\frac{\beta}{d_{1} \mu+\beta d_{3}}\left(\mu y_{1}-d_{3}\right)+1,
$$

which implies that $\Re_{1}<1$ is equivalent to $y_{1}<\frac{d_{3}}{\mu}$. On the other hand, the term $\mu y_{1} / d_{3}$ can be seen as the immune reproductive number, which expresses the average number of activated CTLs generated from one CTLs during its life time $1 / d_{3}$ through the stimulation of the infected cells $y_{1}$. It is reasonable that immune is activated in the case where $\Re_{1}>1$.

We summarize the above analysis in the following result.

Proposition 2.2 If $\Re_{0} \leq 1, E_{0}=\left(\lambda / d_{1}, 0,0\right)$ is the only equilibrium in $\Gamma$. If $\Re_{1} \leq 1<\Re_{0}$, the carrier equilibrium $E_{1}=\left(x_{1}, y_{1}, 0\right)$ appears and is the only chronic-infection equilibrium in $\Gamma$. If $\Re_{1}>1$, both the carrier equilibrium $E_{1}$ and the HAM/TSP equilibrium $E_{2}=\left(x_{2}, y_{2}, z_{2}\right)$ appear. 


\section{Dynamics of system (1.2) for $\Re_{1} \leq 1$}

In what follows, we investigate the dynamics of system (1.2) when $\Re_{1} \leq 1$. We begin by using the inequality $g(x)=x-1-\ln x \geq g(1)=0$ with equality holding if and only if $x=1$, which can simplify many of the expressions in the following calculations. Note that $g$ : $\mathbb{R}^{+} \rightarrow \mathbb{R}^{+}$has strict global minimum $g(1)=0$.

We have the following theorem.

Theorem 3.1 Let $\Re_{0}$ and $\Re_{1}$ be as defined in (2.6) and (2.7). Then, for $\tau>0$ and $\omega>0$, the following statements hold for system (1.2).

(i) If $\Re_{0} \leq 1$, then $E_{0}$ is globally asymptotically stable.

(ii) If $\Re_{1} \leq 1<\Re_{0}$, then $E_{1}$ is globally asymptotically stable.

Proof (i) Define a Lyapunov functional

$$
W_{1}=e^{-d \tau} x_{0} g\left(\frac{x(t)}{x_{0}}\right)+y(t)+\frac{\gamma}{\mu} z(t)+\beta e^{-d \tau} \int_{0}^{\tau} x(t-\theta) y(t-\theta) d \theta+\gamma \int_{0}^{\omega} y(t-\xi) z(t) d \xi .
$$

Using $\lambda=d_{1} x_{0}$ and calculating the time derivative of $W_{1}$ along the solutions of (1.2), we obtain

$$
\begin{aligned}
\left.W_{1}^{\prime}\right|_{(1.2)}= & e^{-d \tau}\left(1-\frac{x_{0}}{x(t)}\right) x^{\prime}(t)+y^{\prime}(t)+\frac{\gamma}{\mu} z^{\prime}(t)+\beta e^{-d \tau}[x(t) y(t)-x(t-\tau) y(t-\tau)] \\
& +\gamma(y(t) z(t)-y(t-\omega) z(t)) \\
= & -\frac{d}{x} e^{-d \tau}\left(x(t)-x_{0}\right)^{2}+\left(\beta e^{-d \tau} \frac{\lambda}{d_{1}}-d_{2}\right) y(t)-\frac{\gamma d_{3}}{\mu} z(t) \\
= & -\frac{d}{x} e^{-d \tau}\left(x(t)-x_{0}\right)^{2}+d_{2}\left(\Re_{0}-1\right) y(t)-\frac{\gamma d_{3}}{\mu} z(t) .
\end{aligned}
$$

Therefore, $\Re_{0} \leq 1$ ensures that $\left.W_{1}^{\prime}\right|_{(1.2)} \leq 0$ for all $x \geq 0, y \geq 0, z(t) \geq 0$, and $\left.W_{1}^{\prime}\right|_{(1.2)}=0$ if and only if $x(t)=x_{0}, y(t)=0, z(t)=0$ for $\Re_{0}<1$ or $x(t)=x_{0}, z(t)=0$ for $\Re_{0}=1$. For the both cases, it is easy to show that the largest invariant set in $\left\{(x, y, v)\left|W_{1}^{\prime}\right|_{(1.2)}=0\right\}$ is $\left\{E_{0}\right\}$. LaSalle's invariance principle (Theorem 2.5.3 of Kuang [20]) shows that $E_{0}$ is globally asymptotically stable when $\Re_{0} \leq 1$.

(ii) Define a Lyapunov functional

$$
\begin{aligned}
W_{2}= & e^{-d \tau} x_{1} g\left(\frac{x(t)}{x_{1}}\right)+y_{1} g\left(\frac{y(t)}{y_{1}}\right)+\frac{\gamma}{\mu} z(t) \\
& +d_{2} y_{1} \int_{0}^{\tau} g\left(\frac{x(t-\theta) y(t-\theta)}{x_{1} y_{1}}\right) d \theta+\gamma \int_{0}^{\omega} y(t-\xi) z(t) d \xi .
\end{aligned}
$$

Calculating the time derivative of $W_{2}$ along the solution of (1.2), we obtain

$$
\begin{aligned}
\left.W_{2}^{\prime}\right|_{(1.2)}= & e^{-d \tau}\left(1-\frac{x_{1}}{x(t)}\right) x^{\prime}(t)+\left(1-\frac{y_{1}}{y(t)}\right) y^{\prime}(t) \\
& +\frac{\gamma}{\mu} z^{\prime}(t)+\beta e^{-d \tau}[x(t) y(t)-x(t-\tau) y(t-\tau)] \\
& +d_{2} y_{1} \ln \frac{x(t-\tau) y(t-\tau)}{x(t) y(t)}+\gamma(y(t) z(t)-y(t-\omega) z(t))
\end{aligned}
$$




$$
\begin{aligned}
= & -\frac{d}{x} e^{-d \tau}\left(x(t)-x_{1}\right)^{2}+d_{2} y_{1}\left(1-\frac{x_{1}}{x(t)}+\ln \frac{x_{1}}{x(t)}\right) \\
& +d_{2} y_{1}\left(1-\frac{x(t-\tau) y(t-\tau)}{x_{1} y(t)}+\ln \frac{x(t-\tau) y(t-\tau)}{x_{1} y(t)}\right)+\gamma z(t)\left(y_{1}-\frac{d_{3}}{\mu}\right) .
\end{aligned}
$$

Here we used the facts that $\lambda=d_{1} x_{1}+\beta x_{1} y_{1}$ and $d_{2}=\beta e^{-d \tau} x_{1}$. Therefore, $\Re_{1} \leq 1$ ensures that $\left.W_{2}^{\prime}\right|_{(1.2)} \leq 0$ for all $x \geq 0, y \geq 0, z(t) \geq 0$, and $\left.W_{2}^{\prime}\right|_{(1.2)}=0$ if and only if $x(t)=x_{1}, y(t)=y_{1}$, $z(t)=0$ for $\Re_{1}<1$ or $x(t)=x_{1}, y(t)=y_{1}$ for $\Re_{1}=1$. For the both cases, it is easy to show that the largest invariant set in $\left\{(x, y, z)\left|W_{2}^{\prime}\right|_{(1.2)}=0\right\}$ is $\left\{E_{1}\right\}$. LaSalle's invariance principle (Theorem 2.5 .3 of Kuang [20]) shows that $E_{1}$ is globally asymptotically stable when $\Re_{1} \leq$ $1<\Re_{0}$.

Remark 3.1 Theorem 3.1 gives a complete picture of global dynamics of system (1.2) for the case where $\Re_{1} \leq 1$. It is shown that $\Re_{0}$ and $\Re_{1}$ as two sharp threshold parameters together determine the outcomes of the HTLV-I infection: when $\Re_{0} \leq 1$, then the HTLV-I viruses are cleared; when $\Re_{1} \leq 1<\Re_{0}$, then HTLV-I infection becomes chronic with no persistent CTLs immune response. The patient remains as an asymptotic carrier.

\section{Dynamics of system (1.2) when $\Re_{1}>1$}

From Proposition 2.2, when $\Re_{1}>1$, the HAM/TSP equilibrium $E_{2}=\left(x_{2}, y_{2}, z_{2}\right)$ appears and both HTLV-I infection and CTLs immune response will persist. The patient has a high risk to developing HAM/TSP. In this section, we will focus on the stability of the HAM/TSP equilibrium $E_{2}$.

We first consider the infection process without immune activation delay $\omega$ but with intracellular delay $\tau$. In this case, system (1.2) will reduce to the following system of delay differential equations:

$$
\left\{\begin{array}{l}
x^{\prime}(t)=\lambda-d_{1} x(t)-\beta x(t) y(t), \\
y^{\prime}(t)=\beta e^{-d \tau} x(t-\tau) y(t-\tau)-d_{2} y(t)-\gamma y(t) z(t), \\
z^{\prime}(t)=\mu y(t) z(t)-d_{3} z(t),
\end{array}\right.
$$

associated with initial conditions

$$
\varphi=\left(\varphi_{1}, \varphi_{2}, \varphi_{3}\right) \in \mathcal{C}^{+}, \quad \varphi(0)>0 .
$$

Based on Theorem 3.1, it is easy to see that the infection-free equilibrium $E_{0}$ and immune-free equilibrium $E_{1}$ of (4.1) (remains the same as the ones in (1.2)) can be proved to be globally asymptotically stable by using the Lyapunov functionals $W_{1}$ and $W_{2}$ when $\omega=0$. Furthermore, using similar arguments to Theorem 3.1, combining the equations $\lambda=d_{1} x_{2}+\beta x_{2} y_{2}, \beta e^{-d \tau} x_{2} y_{2}=d_{2} y_{2}+\gamma y_{2} z_{2}$ and $\mu y_{2}=d_{3}$, we can prove that HAM/TSP equilibrium $E_{2}$ of (4.1) is globally asymptotically stable by using a Lyapunov functional

$$
\begin{aligned}
W_{3}= & e^{-d \tau} x_{2} g\left(\frac{x(t)}{x_{2}}\right)+y_{2} g\left(\frac{y(t)}{y_{2}}\right)+\frac{\gamma}{\mu} z_{2} g\left(\frac{z(t)}{z_{2}}\right) \\
& +\left(d_{2} y_{2}+\gamma y_{2} z_{2}\right) \int_{0}^{\tau} g\left(\frac{x(t-\theta) y(t-\theta)}{x_{2} y_{2}}\right) d \theta .
\end{aligned}
$$

Hence we arrive at the following theorem on the global dynamics of (4.1) with (4.2). 
Theorem 4.1 Let $\Re_{0}$ and $\Re_{1}$ be as defined in (2.6) and (2.7), the following statements hold for system (4.1).

(i) If $\Re_{0} \leq 1$, then the infection-free equilibrium $E_{0}\left(x_{0}, 0,0\right)$ is globally asymptotically stable;

(ii) If $\Re_{1} \leq 1<\Re_{0}$, then the immune-free equilibrium $E_{1}\left(x_{1}, y_{1}, 0\right)$ is globally asymptotically stable;

(iii) If $\Re_{1}>1$, then the HAM/TSP equilibrium $E_{2}\left(x_{2}, y_{2}, z_{2}\right)$ is globally asymptotically stable.

Remark 4.1 As to the main results in Song et al. [21], Theorem 4.1 gives a confirmative answer that there are no sustained oscillations to occur when the immune response is not incorporated with time delay.

From Theorem 4.1 we know that the intracellular delay $\tau$ does not affect the stability of the HAM/TSP equilibrium $E_{2}$ besides the numeric values of $E_{2}$ and the basic reproductive numbers. In order to discuss the effects of immune activation delay $\omega$, for mathematical tractability, we next just consider the case $\tau=0$ and $\omega>0$ without losing the major biological feature. In this case, system (1.2) will reduce to the following system of delay differential equations:

$$
\left\{\begin{array}{l}
x^{\prime}(t)=\lambda-d_{1} x(t)-\beta x(t) y(t) \\
y^{\prime}(t)=\beta x(t) y(t)-d_{2} y(t)-\gamma y(t) z(t) \\
z^{\prime}(t)=\mu y(t-\omega) z(t)-d_{3} z(t)
\end{array}\right.
$$

Now the basic reproductive number and the immune reproductive number of (4.3) should be written by

$$
\Re_{0}^{*}=\frac{\lambda \beta}{d_{1} d_{2}}, \quad \text { and } \quad \Re_{1}^{*}=\frac{\lambda \beta \mu}{d_{1} d_{2} \mu+\beta d_{2} d_{3}},
$$

and $E^{*}$ is denoted as the interior equilibrium for DDEs models (4.3). In the following, we shall show that the time delay $\omega$ can destabilize $E^{*}$ and leads to Hopf bifurcations. Concretely, using the normal form theory and center manifold argument, we will give the explicit formulae which determine the stability and direction of bifurcated periodic solutions.

Translating $E^{*}$ to the origin through a change of variables $x_{2}(t)=x(t)-x^{*}, y_{2}(t)=y(t)-y^{*}$, $z_{2}(t)=z(t)-z^{*}$, system (4.3) becomes

$$
\left\{\begin{array}{l}
x_{2}^{\prime}(t)=-\left(\beta y^{*}+d_{1}\right) x_{2}(t)-\beta x^{*} y_{2}(t)-\beta x_{2}(t) y_{2}(t) \\
y_{2}^{\prime}(t)=\beta x_{2}(t) y_{2}(t)+\beta y^{*} x_{2}(t)-\gamma y^{*} z_{2}(t)-\gamma y_{2}(t) z_{2}(t) \\
z_{2}^{\prime}(t)=\mu y_{2}(t-\omega) z_{2}(t)+\mu z^{*} y_{2}(t-\omega) .
\end{array}\right.
$$

The characteristic equation associated with the linearization of system $(4.3)$ at $(0,0,0)$ is

$$
\operatorname{det}\left(\begin{array}{ccc}
\xi+\beta y^{*}+d_{1} & \beta x^{*} & 0 \\
-\beta y^{*} & \xi & \gamma y^{*} \\
0 & -\mu z^{*} e^{-\xi \omega} & \xi
\end{array}\right)=0 .
$$


We obtain

$$
\xi^{3}+a_{1} \xi^{2}+b_{1} \xi+\left(c_{1} \xi+c_{0}\right) e^{-\xi \omega}=0
$$

where

$$
a_{1}=\beta y^{*}+d_{1}, \quad b_{1}=\beta^{2} x^{*} y^{*}, \quad c_{0}=\left(\beta y^{*}+d_{1}\right) \gamma d_{3} z^{*}, \quad c_{1}=\gamma d_{3} z^{*} .
$$

When $\omega=0$, the characteristic equation is calculated as follows:

$$
\xi^{3}+a_{1} \xi^{2}+\left(b_{1}+c_{1}\right) \xi+c_{0}=0 .
$$

By the Routh-Hurwitz criterion we know that

$$
\begin{aligned}
& a_{1}>0, \quad c_{0}>0, \\
& \begin{aligned}
a_{1}\left(b_{1}+c_{1}\right)-c_{0} & =\left(\beta y^{*}+d_{1}\right)\left(\beta^{2} x^{*} y^{*}+\gamma d_{3} z^{*}\right)-\left(\beta y^{*}+d_{1}\right) \gamma d_{3} z^{*} \\
& =\beta^{3} x^{*}\left(y^{*}\right)^{2}+\beta^{2} d_{1} x^{*} y^{*}>0,
\end{aligned}
\end{aligned}
$$

then all roots of (4.6) have negative real parts.

Next, we will use the delay $\omega>0$ as a bifurcation parameter and investigate stability changes at the HAM/TSP equilibrium $E^{*}$. We will show that a Hopf bifurcation occurs for an open set of parameter values. This rigorously establishes that periodic oscillations exist in system (4.3).

Obviously, $\xi=0$ is not a root of (4.5) and $\xi=i p$ is a root of (4.5) if and only if

$$
-p^{3} i-a_{1} p^{2}+b_{1} p i+\left(i c_{1} p+c_{0}\right)(\cos p \omega-i \sin p \omega)=0 .
$$

Separating the real and imaginary parts gives

$$
\left\{\begin{array}{l}
-p^{3}+b_{1} p=c_{0} \sin p \omega-c_{1} p \cos p \omega \\
a_{1} p^{2}=c_{0} \cos p \omega+c_{1} p \sin p \omega
\end{array}\right.
$$

Squaring and adding both equations of (4.7) lead to

$$
F(p)=p^{6}+\left(a_{1}^{2}-2 b_{1}\right) p^{4}+\left(b_{1}^{2}-c_{1}^{2}\right) p^{2}-c_{0}^{2}=0 .
$$

Let $u=p^{2}$, then it follows that

$$
G(u)=u^{3}+\left(a_{1}^{2}-2 b_{1}\right) u^{2}+\left(b_{1}^{2}-c_{1}^{2}\right) u-c_{0}^{2}=0
$$

has a positive root $u=p^{2}$ since $i p$ is a purely imaginary root of (4.5). Note that

$$
G^{\prime}(u)=3 u^{2}+2\left(a_{1}^{2}-2 b_{1}\right) u+\left(b_{1}^{2}-c_{1}^{2}\right) .
$$

Let

$$
\Delta=\left(a_{1}^{2}-2 b_{1}\right)^{2}-3\left(b_{1}^{2}-c_{1}^{2}\right)
$$


Then

(1) If $\Delta \leq 0$, then $G^{\prime}(u) \geq 0$, and thus $G(u)$ is monotonically increasing. Therefore, by $-c_{0}^{2}=G(0)<0$ and $\lim _{u \rightarrow \infty} G(u)=\infty$, we know that (4.9) has at least one positive root, and characteristic roots can cross the imaginary axis.

(2) If $\Delta>0$, then the graph of $G(u)$ has critical points

$$
u^{*}=\frac{-\left(a_{1}^{2}-2 b_{1}\right)+\sqrt{\Delta}}{3}, \quad u^{* *}=\frac{-\left(a_{1}^{2}-2 b_{1}\right)-\sqrt{\Delta}}{3} .
$$

Clearly, $G^{\prime \prime}\left(u^{*}\right)=2 \sqrt{\Delta}>0$ and $G^{\prime \prime}\left(u^{* *}\right)=-2 \sqrt{\Delta}<0$, it follows that $u^{*}$ and $u^{* *}$ are the local minimum and the local maximum of $G(u)$, respectively.

If $u^{*}>0$ and $G\left(u^{*}\right)<0$, then $G(u)=0$ has positive roots. Without loss of generality, let $u_{k}, 1 \leq k \leq 3$, be the three positive roots of $G(u)=0$, respectively. Then $F(p)=0$ has three positive roots $p_{k}=\sqrt{u_{k}}, 1 \leq k \leq 3$. Solving (4.7) for $\omega$ yields

$$
\cos p_{k} \omega=\frac{c_{0} a_{1} p_{k}^{2}+c_{1} p_{k}^{4}-b_{1} c_{1} p_{k}^{2}}{c_{0}^{2}+c_{1}^{2} p_{k}^{2}}
$$

Denote

$$
\omega_{k}^{(j)}=\frac{1}{p_{k}}\left[\arccos \frac{c_{0} a_{1} p_{k}^{2}+c_{1} p_{k}^{4}-b_{1} c_{1} p_{k}^{2}}{c_{0}^{2}+c_{1}^{2} p_{k}^{2}}+2 j \pi\right],
$$

where $1 \leq k \leq 3, j=0,1,2 \ldots$. Then $\pm i p_{k}$ is a pair of purely imaginary roots of (4.5) with $\omega=\omega_{k}^{(j)}$.

Define

$$
\begin{aligned}
& \omega_{0}=\omega_{k}=\min _{1 \leq i \leq 3}\left\{\omega_{i} \mid u_{i} \text { is a positive solution of } G(u)=0\right\}, \\
& p_{0}=p_{k} .
\end{aligned}
$$

Then $\omega_{0}$ is the first value of $\omega$ when a pair of characteristic roots cross the imaginary axis at $\pm i p_{0}$. We thus obtain the following result.

Theorem 4.2 If $\Delta>0, u^{*}=\frac{-\left(a_{1}^{2}-2 b_{1}\right)+\sqrt{\Delta}}{3}>0$ and $G\left(u^{*}\right)<0$, then there exist $\omega_{0}>0$ and $p_{0}$ as in (4.13) such that the HAM/TSP equilibrium $E^{*}$ is asymptotically stable for $\omega \in\left[0, \omega_{0}\right)$. Furthermore, if $G^{\prime}\left(p_{0}^{2}\right) \neq 0$, then system (4.3) undergoes a Hopfbifurcation at the HAM/TSP equilibrium $E^{*}$ when $\omega=\omega_{0}$.

Proof It remains to show that the transversality condition for the Hopf bifurcation holds at $\omega=\omega_{0}$. Differentiating (4.6) with respect to $\omega$ gives

$$
\left[\xi^{\prime}(\omega)\right]^{-1}=\frac{\left[3 \xi^{2}+2 a_{1} \xi+b_{1}\right] e^{\xi \omega}}{\xi\left(c_{1} \xi+c_{0}\right)}+\frac{c_{1}}{\xi\left(c_{1} \xi+c_{0}\right)}-\frac{\omega}{\xi} .
$$

Using (4.5) we obtain

$$
\begin{aligned}
{\left[(\operatorname{Re} \xi(\omega))^{\prime}\right]_{\omega=\omega_{0}}^{-1} } & =\operatorname{Re}\left[\frac{\left[3 \xi^{2}+2 a_{1} \xi+b_{1}\right] e^{\xi \omega}}{\xi\left(c_{1} \xi+c_{0}\right)}\right]_{\omega=\omega_{0}}+\operatorname{Re}\left[\frac{c_{1}}{\xi\left(c_{1} \xi+c_{0}\right)}\right]_{\omega=\omega_{0}} \\
& =\frac{1}{p_{0}^{2}\left(c_{1}^{2} p_{0}^{2}+c_{0}^{2}\right)}\left[\left(b_{1}-3 p_{0}^{2}\right) p_{0}\left(c_{0} \sin p_{0} \omega_{0}-c_{1} p_{0} \cos p_{0} \omega_{0}\right)\right.
\end{aligned}
$$




$$
\begin{aligned}
& \left.+2 a_{1} p_{0}^{2}\left(c_{0} \cos p_{0} \omega_{0}+c_{1} p_{0} \sin p_{0} \omega_{0}\right)-c_{1}^{2} p_{0}^{2}\right] \\
= & \frac{3 p_{0}^{6}+2\left(a_{1}^{2}-2 b_{1}\right) p_{0}^{4}+\left(b_{1}^{2}-c_{1}^{2}\right) p_{0}^{2}}{p_{0}^{4} c_{1}^{2}+p_{0}^{2} c_{0}^{2}} \\
= & \frac{G^{\prime}\left(p_{0}^{2}\right)}{c_{1}^{2} p_{0}^{2}+c_{0}^{2}} .
\end{aligned}
$$

Therefore, $\operatorname{sign}\left[(\operatorname{Re} \xi(\omega))^{\prime}\right]_{\omega=\omega_{0}}=\operatorname{sign} G^{\prime}\left(p_{0}^{2}\right)$. Thus, if $G^{\prime}\left(p_{0}^{2}\right) \neq 0$, the transversality condition holds and a Hopf bifurcation occurs at $\omega=\omega_{0}$. This completes the proof.

We have identified parameter regimes in which delay $\omega$ can destabilize the HAM/TSP equilibrium and lead to a Hopf bifurcation. This shows that when $\omega>\omega_{0}$ and is close to $\omega_{0}$, periodic solutions exist. As a consequence, when HAM/TSP develops, the CD4 ${ }^{+}$count, proviral load, and the HTLV-I specific CTLs frequency can oscillate around the equilibrium level. Next, using techniques from normal form and center manifold theory (see, e.g., Hassard et al. [22]), we study the direction of the Hopf bifurcation and the stability of the bifurcating periodic solutions when $\omega=\omega_{0}$.

Let $\omega=\omega_{0}+\mu$, and we use $\mu$ as the bifurcation parameter with $\mu=0$ the Hopf bifurcation value. We first scale the time $t \mapsto t / \omega$ in system (4.3) and set

$$
B_{1}=\left(\begin{array}{ccc}
-\left(\beta y^{*}+d_{1}\right) & -\beta x^{*} & 0 \\
\beta y^{*} & 0 & -\gamma y^{*} \\
0 & 0 & 0
\end{array}\right), \quad B_{2}=\left(\begin{array}{ccc}
0 & 0 & 0 \\
0 & 0 & 0 \\
0 & \mu z^{*} & 0
\end{array}\right)
$$

Define an operator $L_{\mu}: C\left([-1,0], \mathbb{R}^{3}\right) \rightarrow \mathbb{R}$ as

$$
L_{\mu}(\phi)=\left(\omega_{0}+\mu\right) B_{1} \phi(0)+\left(\omega_{0}+\mu\right) B_{2} \phi(-1)
$$

with

$$
f(\mu, \phi)=\left(\omega_{0}+\mu\right)\left(\begin{array}{c}
-\beta \phi_{1}(0) \phi_{2}(0) \\
\beta \phi_{1}(0) \phi_{2}(0)-\gamma \phi_{2}(0) \phi_{3}(0) \\
\mu \phi_{2}(-1) \phi_{3}(0)
\end{array}\right)
$$

for $\phi=\left(\phi_{1}, \phi_{2}, \phi_{3}\right)^{T} \in C\left([-1,0], \mathbb{R}^{3}\right)$. We can rewrite system (4.3) as an abstract FDE in $C\left([-1,0], \mathbb{R}^{3}\right)$

$$
v^{\prime}(t)=L_{\mu}\left(v_{t}\right)+f\left(\mu, v_{t}\right)
$$

where $v_{t}=\left(x_{2}(t), y_{2}(t), z_{2}(t)\right)^{T} \in \mathbb{R}^{3}$. Let

$$
\delta(\theta)= \begin{cases}0, & \theta \neq 0 \\ 1, & \theta=0\end{cases}
$$

and define

$$
\eta(\theta, \mu)=\left(\omega_{0}+\mu\right) B_{1} \delta(\theta)-\left(\omega_{0}+\mu\right) B_{2} \delta(\theta+1) .
$$


Then operator $L_{\mu}$ in (4.16) can be represented in an integral form as

$$
L_{\mu} \phi=\int_{-1}^{0} d \eta(\theta, 0) \phi(\theta), \quad \text { for } \phi \in C\left([-1,0], \mathbb{R}^{3}\right) .
$$

Define operators

$$
A(\mu) \phi(\theta)= \begin{cases}\frac{d \phi(\theta)}{d \theta}, & \theta \in[-1,0) ; \\ \int_{-1}^{0} d \eta(s, \mu) \phi(s), & \theta=0\end{cases}
$$

and

$$
R(\mu) \phi(\theta)= \begin{cases}0, & \theta \in[-1,0) ; \\ f(\mu, \phi), & \theta=0 .\end{cases}
$$

Then system (4.3) is written as an abstract ordinary differential equation in the Banach space $C\left([-1,0], \mathbb{R}^{3}\right)$

$$
v_{t}^{\prime}=A(\mu) v_{t}+R(\mu) v_{t}
$$

For $\psi \in C^{1}\left([0,1],\left(R^{3}\right)^{*}\right)$, define an operator

$$
A^{*} \psi(s)= \begin{cases}-\frac{d \psi(s)}{d s}, & s \in(0,1] \\ \int_{-1}^{0} d \eta^{T}(t, 0) \phi(-t), & s=0\end{cases}
$$

and a bilinear form

$$
\langle\psi(s), \phi(\theta)\rangle=\bar{\psi}(0) \phi(0)-\int_{-1}^{0} \int_{\xi=0}^{\theta} \bar{\psi}(\xi-\theta) d \eta(\theta) \phi(\xi) d \xi
$$

where $\eta(\theta)=\eta(\theta, 0)$. Then $A(0)$ and $A^{*}$ are adjoint operators with respect to this bilinear form. From the previous section, we know that $\pm i p_{0} \omega_{0}$ are eigenvalues of $A(0)$ and therefore they are also eigenvalues of $A^{*}$. Suppose that $q(\theta)=q(0) e^{i p_{0} \omega_{0} \theta}$ is an eigenvector of $A(0)$ corresponding to the eigenvalue $i p_{0} \omega_{0}$. Then $A(0)=i p_{0} \omega_{0} q(\theta)$. When $\theta=0$, we obtain

$$
\left[i p_{0} \omega_{0} I-\int_{-1}^{0} d \eta(\theta) e^{i p_{0} \omega_{0} \theta}\right] q(0)=0
$$

which yields $q(0)=\left(1, q_{2}, q_{3}\right)^{T}$, where

$$
q_{2}=-\frac{i p_{0}+d_{1}+\beta y^{*}}{\beta x^{*}}, \quad q_{3}=\frac{\beta}{\gamma}+\frac{i p_{0}\left(i p_{0}+d_{1}+\beta y^{*}\right)}{\beta \gamma x^{*} y^{*}} .
$$

Similarly, it can be verified that $q^{*}(s)=D\left(1, q_{2}^{*}, q_{3}^{*}\right) e^{i p_{0} \omega_{0} s}$ is the eigenvector of $A^{*}$ corresponding to $-i p_{0} \omega_{0}$, where

$$
q_{2}^{*}=1+\frac{d_{1}-i p_{0}}{\beta y^{*}}, \quad q_{3}^{*}=\frac{\beta^{2} x^{*} y^{*}-i p_{0}\left(\beta y^{*}+d_{1}-i p_{0}\right)}{\beta \mu y^{*} z^{*} e^{i p_{0} \omega_{0}}} .
$$


By (4.17), we get

$$
\begin{aligned}
\left\langle q^{*}(s), q(\theta)\right\rangle= & \bar{D}\left(1, q_{2}^{*}, q_{3}^{*}\right)\left(1, q_{2}, q_{3}\right)^{T} \\
& -\int_{-1}^{0} \int_{\xi=0}^{\theta}\left(1, q_{2}^{*}, q_{3}^{*}\right) e^{-i p_{0} \omega_{0}(\xi-\theta)} d \eta(\theta)\left(1, q_{2}, q_{3}\right)^{T} e^{i p_{0} \omega_{0} \xi} d \xi \\
= & 1+\bar{q}_{2} q_{2}^{*}+\bar{q}_{3} q_{3}^{*}+\mu z^{*} \omega_{0} \bar{q}_{2} q_{3}^{*} e^{i p_{0} \omega_{0}} .
\end{aligned}
$$

We can choose $\bar{D}=\left(1+\bar{q}_{2} q_{2}^{*}+\bar{q}_{3} q_{3}^{*}+\mu z^{*} \omega_{0} \bar{q}_{2} q_{3}^{*} e^{i p_{0} \omega_{0}}\right)^{-1}$ such that $\left\langle q^{*}, q\right\rangle=1$.

Following the algorithms in [22] and using the same notations as there to compute the coordinates describing the center manifold $C_{0}$ at $\mu=0$, we obtain the coefficients:

$$
\begin{aligned}
g_{20}= & 2 \omega_{0} \bar{D} q_{2}\left[\left(q_{2}^{*}-1\right) \beta-\bar{q}_{2}^{*} \gamma q_{3}+\mu q_{3} \bar{q}_{3}^{*} e^{-i p_{0} \omega_{0}}\right], \\
g_{11}= & \omega_{0} \bar{D}\left[\left(q_{2}^{*}-1\right) \beta\left(q_{2}+\bar{q}_{2}\right)-\bar{q}_{2}^{*} \gamma\left(q_{2} \bar{q}_{3}+\bar{q}_{2} q_{3}\right)+\mu \bar{q}_{3}^{*}\left(q_{2} \bar{q}_{3} e^{-i p_{0} \omega_{0}}+\bar{q}_{2} q_{3} e^{i p_{0} \omega_{0}}\right)\right], \\
g_{02}= & 2 \omega_{0} \bar{D} \bar{q}_{2}\left[\left(q_{2}^{*}-1\right) \beta-\bar{q}_{2}^{*} \gamma \bar{q}_{3}+\mu \bar{q}_{3} \bar{q}_{3}^{*} e^{i p_{0} \omega_{0}}\right], \\
g_{21}= & 2 \omega_{0} \bar{D}\left[\left(q_{2}^{*}-1\right) \beta\left(W_{11}^{(2)}(0)+\frac{1}{2} W_{20}^{(2)}(0)+\frac{1}{2} \bar{q}_{2} W_{20}^{(1)}(0)+q_{2} W_{11}^{(1)}(0)\right)\right. \\
& -\bar{q}_{2}^{*} \gamma\left(q_{2} W_{11}^{(3)}(0)+\frac{1}{2} \bar{q}_{2} W_{20}^{(3)}(0)+\frac{1}{2} \bar{q}_{3} W_{20}^{(2)}(0)+q_{3} W_{11}^{(2)}(0)\right) \\
& \left.+\mu \bar{q}_{3}^{*}\left(q_{2} W_{11}^{(3)}(0) e^{-i p_{0} \omega_{0}}+\frac{1}{2} \bar{q}_{2} W_{20}^{(3)}(0) e^{i p_{0} \omega_{0}}+\frac{1}{2} \bar{q}_{3} W_{20}^{(2)}(-1)+q_{3} W_{11}^{(2)}(-1)\right)\right],
\end{aligned}
$$

where $\theta \in[-1,0]$. We still need to compute $W_{20}(\theta)$ and $W_{11}(\theta)$.

$$
\begin{aligned}
& W_{20}(\theta)=\frac{i g_{20}}{p_{0} \omega_{0}} q(0) e^{i p_{0} \omega_{0} \theta}+\frac{i \bar{g}_{02}}{3 p_{0} \omega_{0}} \bar{q}(0) e^{-i p_{0} \omega_{0} \theta}+E_{1} e^{2 i p_{0} \omega_{0} \theta}, \\
& W_{11}(\theta)=-\frac{i g_{11}}{p_{0} \omega_{0}} q(0) e^{i p_{0} \omega_{0} \theta}+\frac{i \bar{g}_{11}}{p_{0} \omega_{0}} \bar{q}(0) e^{-i p_{0} \omega_{0} \theta}+E_{2}
\end{aligned}
$$

where

$$
\begin{aligned}
& E_{1}=\left(\begin{array}{ccc}
2 i p_{0}+\beta y^{*}+d_{1} & \beta x^{*} & 0 \\
-\beta y^{*} & 2 i p_{0} & \gamma y^{*} \\
0 & -\mu z^{*} e^{-2 i p_{0} \omega_{0}} & 2 i p_{0}-\mu y^{*}+d_{3}
\end{array}\right)^{-1}\left(\begin{array}{c}
-\beta q_{2} \\
\beta q_{2}-\gamma q_{2} q_{3} \\
\mu q_{2} q_{3} e^{-i p_{0} \omega_{0}}
\end{array}\right), \\
& E_{2}=\left(\begin{array}{ccc}
-\left(\beta y^{*}+d_{1}\right) & -\beta x^{*} & 0 \\
\beta y^{*} & 0 & -\gamma y^{*} \\
0 & \mu z^{*} & \mu y^{*}-d_{3}
\end{array}\right)^{-1}\left(\begin{array}{c}
-\beta\left(q_{2}+\bar{q}_{2}\right) \\
\gamma\left(q_{2} \bar{q}_{3}+\bar{q}_{2} q_{3}\right)-\beta\left(q_{2}+\bar{q}_{2}\right) \\
\mu\left(q_{3} \bar{q}_{2} e^{i p_{0} \omega_{0}}+q_{2} \bar{q}_{3} e^{-i p_{0} \omega_{0}}\right)
\end{array}\right) .
\end{aligned}
$$

And consequently $g_{21}$ can be expressed explicitly, and we can compute the following quantities:

$$
\begin{aligned}
& c_{1}(0)=\frac{i}{2 p_{0} \omega_{0}}\left[g_{21} g_{20}-2\left|g_{11}\right|^{2}-\frac{\left|g_{02}\right|^{2}}{3}\right]+\frac{g_{21}}{2}, \\
& \mu_{2}=\frac{\operatorname{Re}\left(c_{1}(0)\right)}{\operatorname{Re}\left(\xi^{\prime}\left(p_{0}\right)\right)} \\
& \beta_{2}=2 \operatorname{Re}\left(c_{1}(0)\right) .
\end{aligned}
$$


Table 1 Parameter values of system (4.3) used in Section 5

\begin{tabular}{lcl}
\hline Parameter & Value & Unit \\
\hline$\lambda$ & 160 & day $^{-1} \mathrm{~mm}^{-3}$ \\
$\beta$ & 0.002 & day $^{-1} \mathrm{~mm}^{-3}$ \\
$\mu$ & 0.2 & day $^{-1}$ \\
$\gamma$ & 0.2 & day $^{-1}$ \\
$d_{1}$ & 0.16 & day $^{-1}$ \\
$d_{2}$ & 1.85 & day $^{-1}$ \\
$d_{3}$ & 0.8 & day $^{-1}$ \\
\hline
\end{tabular}

By the result of Hassard et al. [22], we have the following theorem.

Theorem 4.3 $\mu_{2}$ determines the direction of the Hopf bifurcation: if $\mu_{2}>0\left(\mu_{2}<0\right)$, then the Hopf bifurcation is supercritical (subcritical) and the bifurcating periodic solutions exist for $\omega>\omega_{0}\left(\omega<\omega_{0}\right) ; \beta_{2}>0\left(\beta_{2}<0\right)$ determines the stability of bifurcating periodic solutions: the bifurcating periodic solutions are orbitally asymptotically stable (unstable) if $\omega>\omega_{0}$ $\left(\omega<\omega_{0}\right)$.

\section{Numerical simulation}

In this section, we further investigate the dynamical behaviors of (4.3) through numerical simulations with the aid of MATHEMATICA. It is shown that the parameter values can be chosen simply to establish the possibility for oscillations in the viral load and $\mathrm{T}$ cell populations.

As in [23, 24], time is measured in days and $x, y, z$ have units $\mathrm{mm}^{-3}$. In the absence of infection, the rate of production of healthy $\mathrm{CD}^{+}{ }^{+} \mathrm{T}$ cells from the bone marrow falls in the range of $100-1,500$ cells $/ \mathrm{mm}^{3} /$ day $[23,24]$, and all three populations considered in our model display natural death rates between 0.001-5/day.

We set the same values of different parameters as in [15] and [21], which is referred in Table 1. After a simple algebraic calculation, it can be verified that the conditions of Theorem 4.2 are satisfied. We can compute that $\omega_{0}=0.6974$. The HAM/TSP equilibrium $E^{*}$ is $E^{*}(952.381,4,3.0282), \mathfrak{R}_{0}^{*}=1.08108$ and $\mathfrak{R}_{1}^{*}=1.0296$. From the analysis in Section 4 and Theorem 4.2, immune activation delay $\omega$ gives more complex behaviors to the dynamics of (4.3): the HAM/TSP equilibrium $E^{*}$ is locally asymptotically stable when $\omega<\omega_{0}$, and a Hopf bifurcation occurs at $\omega=\omega_{0}$; a periodic solution exists when $\omega>\omega_{0}$. Furthermore, we compute $c_{1}(0)=0.0286-7.5081 i$. Therefore $\operatorname{Re}\left(c_{1}(0)\right)>0$ on the center manifold. By Theorem 4.3, we know that the Hopf bifurcation of system (4.3) at the HAM/TSP equilibrium $E^{*}$ is supercritical and the bifurcating periodic solutions are orbitally asymptotically stable. We fix $\omega_{i}=0.05 ; 0.3 ; 0.7 ; 0.9(i=1,2,3,4)$. Figure 1 illustrates that a Hopf bifurcation occurs from the HAM/TSP equilibrium for (4.3). Furthermore, from Figure 1(c) and (d), we can find that there is a growing amplitude along with the increase of immune activation delay.

\section{Conclusion and discussion}

In this paper, we have investigated the dynamics of an HTLV-I model which incorporates intracellular delay and immune activation delay based on the rigorous mathematical analysis. Using two threshold parameters $\Re_{0}$ and $\Re_{1}$, named as the basic reproduction number for viral persistence and for CTLs response, the global dynamics of the proposed system can be obtained by constructing suitable Lyapunov functionals under LaSalle's invariance 

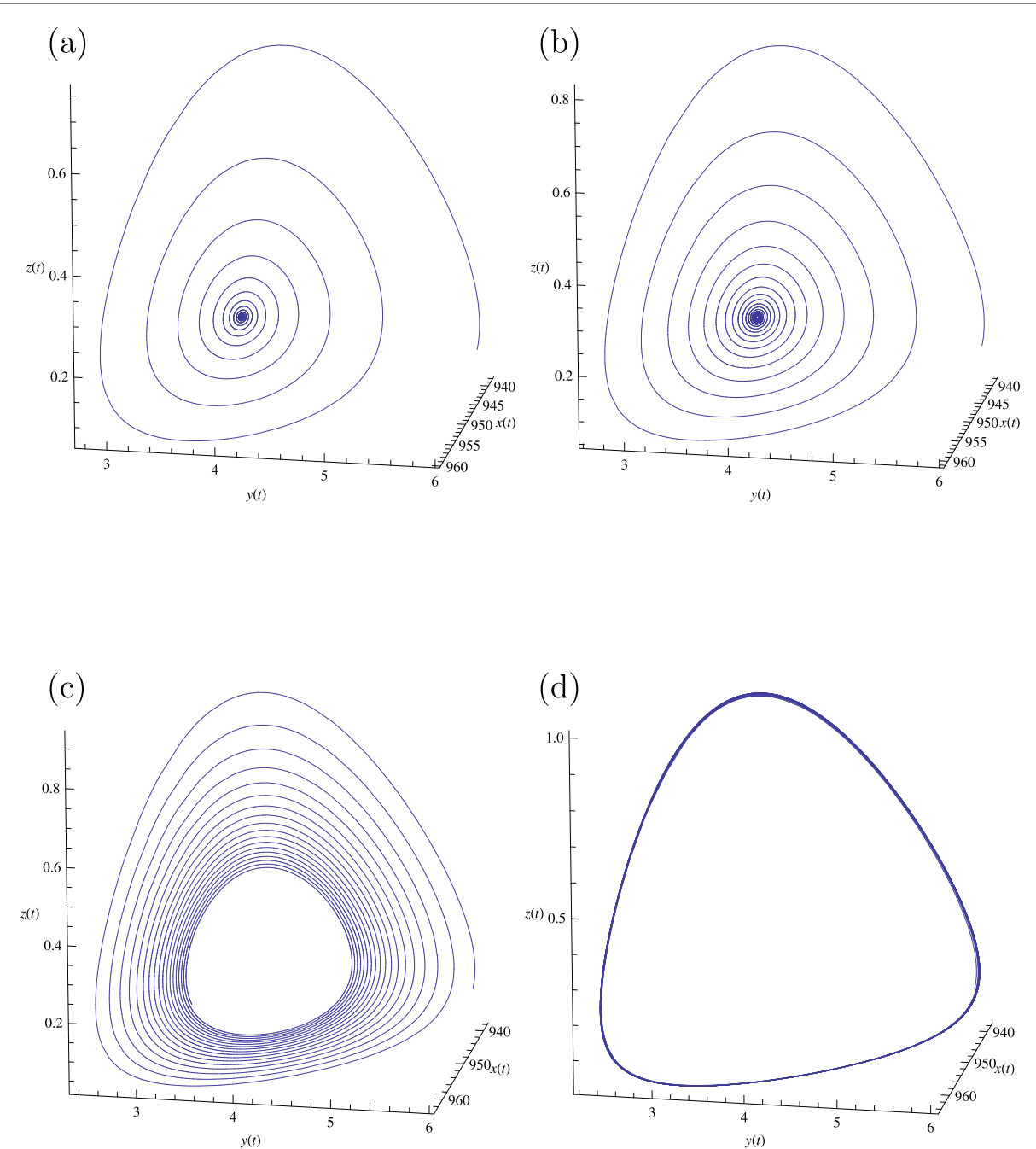

Figure 1 Behavior and phase portrait of system (4.3) with values as in Table 1. $\omega=0.05$ in (a); $\omega=0.3$ in (b); $\omega=0.7$ in (c); $\omega=0.9$ in (d). By Theorem 4.2, we know that a Hopf bifurcation occurs from the HAM/TSP equilibrium. The initial value is $(942,5.97,0.15)$.

principle. Our results show that the intracellular delay $\tau$ does not affect the stability of the HAM/TSP equilibrium, but the immune activation delay can destabilize the equilibrium and lead to a Hopf bifurcation.

As to the previous studies, Wodarz et al. [5], Li and Shu [15, 25], Song et al. [21], Wang et al. [9], Canabarro et al. [16], our results are the first to establish the existence of a Hopf bifurcation in a HTLV-I model with delayed immune response $\left(z^{\prime}(t)=c y(t-\omega) z(t)-b z\right)$ using rigorous mathematical analysis. On the other hand, the results in the present paper (combined with the results in [25] and [15]) show that a mitosis component is not required for sustained oscillations to occur when the immune response is incorporated into the model. These results together have largely enriched our understanding of the effects of intracellular delays in dynamics of viral infection and its interaction with the CTLs immune response. Further, numerical simulation in Figure 1 reveals that the stability switch occurs at critical value of $\omega_{0}$, and the periodic solutions exist with an increasing amplitude when $\omega$ continues to increase. 
HTLV-I infection is rarely cleared from the body. The dynamical outcomes that are biologically relevant are the carrier state and HAM/TSP state. Furthermore, considering that the large number of HTLV-I infected patients are asymptomatic carriers, it is a realistic control and treatment strategy in preventing carriers from developing HAM/TSP by keeping the threshold parameter $\mathfrak{R}_{1}$ below 1 , which may have potential implications for future studies.

\section{Competing interests}

The authors declare that they have no competing interests.

\section{Authors' contributions}

The authors have equal contributions to each part of this paper. All the authors read and approved the final manuscript.

\section{Author details}

${ }^{1}$ School of Mathematical Science, Heilongjiang University, Harbin, 150080, P.R. China. ${ }^{2}$ School of Biomedical Engineering, Third Military Medical University, Chongqing, 400038, P.R. China. ${ }^{3}$ Department of Mathematics and Mechanics, University of Science and Technology Beijing, Beijing, 100083, P.R. China.

\section{Acknowledgements}

The authors would like to thank the anonymous referees and the editor for very helpful suggestions and comments which led to improvements of our original paper. JW was supported by National Natural Science Foundation of China (Nos. 11401182 and 11471089), Natural Science Foundation of Heilongjiang Province (No. A201415), Science and Technology Innovation Team in Higher Education Institutions of Heilongjiang Province (No. 2014TD005), Overseas Studies of Heilongjiang Education Departments (2014), Youth Foundation of Heilongjiang University, project funded by China Post-doctoral Science Foundation (No. 2014M552295) and project funded by Chongqing Postdoctoral Foundation (No. Xm2014024). KW was supported by the National Natural Science Foundation of China (No. 11271369).

Received: 8 February 2015 Accepted: 18 July 2015 Published online: 06 August 2015

\section{References}

1. Gomez-Acevedo, H, Li, MY, Jacobson, S: Multi-stability in a model for CTL response to HTLV-I infection and its consequences in HAM/TSP development and prevention. Bull. Math. Biol. 72, 681-696 (2010)

2. Gessain, A, Barin, F, Vernant, JC, Gout, O, Maurs, L, Calender, A, de Thé, G: Antibodies to human T-lymphotropic virus type-l in patient with tropical spastic paraparesis. Lancet 2, 407-410 (1985)

3. Osame, M, Usuku, K, Izumo, S, ljichi, N, Aminati, H, Igata, A, Matsumoto, M, Tara, M: HTLV-I-associated myelopathy: a new clinical entity. Lancet 1, 1031-1032 (1986)

4. Nowak, MA, May, RM: Virus Dynamics: Mathematical Principles of Immunology and Virology. Cambridge University Press, Cambridge (2000)

5. Wodarz, D, Bangham, CRM: Evolutionary dynamics of HTLV-I. J. Mol. Evol. 50, 448-455 (2000)

6. Wodarz, D, Nowak, MA, Bangham, CRM: The dynamics of HTLV-I and the CTL response. Immunol. Today 20, 220-227 (1999)

7. Burić, N, Mudrinic, M, Vasović, N: Time delay in a basic model of the immune response. Chaos Solitons Fractals 12 , 483-489 (2001)

8. Lang, J, Li, MY: Stable and transient periodic oscillations in a mathematical model for CTL response to HTLV-I infection. J. Math. Biol. 65, 181-199 (2012)

9. Wang, K, Wang, W, Pang, H, Liu, X: Complex dynamic behavior in a viral model with delayed immune response. Physica D 226, 197-208 (2007)

10. Wang, L, Li, MY, Kirschner, D: Mathematical analysis of the global dynamics of a model for HTLV-I infection and ATL progression. Math. Biosci. 179, 207-217 (2002)

11. Bangham, CRM: The immune response to HTLV-I. Curr. Opin. Immunol. 12, 397-402 (2000)

12. Bangham, CRM: The immune control and cell-to-cell spread of human T-lymphotropic virus type 1. J. Gen. Virol. 84, 3177-3189 (2003)

13. Eshima, N, Tabata, M, Okada, T, Karukaya, S: Population dynamics of HTLV-I infection: a discrete-time mathematical epidemic model approach. Math. Med. Biol. 20, 29-45 (2003)

14. Jacobson, S: Immunopathogenesis of human T cell lymphotropic virus type I-associated neurologic disease. J. Infect. Dis. 186, S187-S192 (2002)

15. Li, MY, Shu, H: Global dynamics of a mathematical model for HTLV-I infection of CD4 ${ }^{+} \mathrm{T}$ cells with delayed CTL response. Nonlinear Anal., Real World Appl. 13, 1080-1092 (2012)

16. Canabarro, AA, Gléria, IM, Lyra, ML: Periodic solutions and chaos in a non-linear model for the delayed cellular immune response. Physica A 342, 234-241 (2004)

17. Li, MY, Shu, H: Multiple stable periodic oscillations in a mathematical model of CTL response to HTLV-I infection. Bull. Math. Biol. 73, 1774-1793 (2011)

18. Sun, X, Wei, J: Global existence of periodic solutions in an infection model. Appl. Math. Lett. 48, 118-123 (2015)

19. Huang, G, Yokoi, H, Takeuchi, Y, Kajiwara, T, Sasaki, T: Impact of intracellular delay, immune activation delay and nonlinear incidence on viral dynamics. Jpn. J. Ind. Appl. Math. 28, 383-411 (2011)

20. Kuang, K: Delay Differential Equations with Applications in Population Dynamics. Academics Press, San Diego (1993)

21. Song, $X$, Wang, S, Dong, J: Stability properties and Hopf bifurcation of a delayed viral infection model with lytic immune response. J. Math. Anal. Appl. 373, 345-355 (2011) 
22. Hassard, BD, Kazarinoff, ND, Wan, YH: Theory and Application of Hopf Bifurcation. Cambridge University Press, Cambridge (1981)

23. de Leenheer, P, Smith, HL: Virus dynamics: a global analysis. SIAM J. Appl. Math. 63, 1313-1327 (2002)

24. Perelson, AS, Kirschner, DE, de Boer, R: Dynamics of HIV infection of CD4 ${ }^{+}$T cell. Math. Biosci. 144, 81-125 (1993)

25. Li, MY, Shu, H: Impact of intracellular delays and target-cell dynamics on in vivo viral infections. SIAM J. Appl. Math. 70 2434-2448 (2010)

Submit your manuscript to a SpringerOpen ${ }^{\circ}$ journal and benefit from:

- Convenient online submission

Rigorous peer review

- Immediate publication on acceptance

- Open access: articles freely available online

- High visibility within the field

- Retaining the copyright to your article 